\title{
Evaluation of Ischemia Modified Albumin IMA as New Predictor in Patients with Newly Thyroid Dysfunction in Type 2 Diabetes Mellitus
}

\author{
Baydaa A. Abed ${ }^{1}$, Sanad B. Al-AAraji ${ }^{1}$, Isam N. Salman ${ }^{2}$ \\ ${ }^{1}$ Asst., Department of Chemistry/College of Science for Women/University of Baghdad/Iraq, \\ ${ }^{2}$ Asst. Prof., F.R.C.(u.k), C.A,B.M, National Diabetes Center./Mustansiriyah University/Iraq
}

\begin{abstract}
In the general population Thyroid dysfunctions are common after DM as the most well-known situation to influence the endocrine system. Diabetic patients are at increased danger of thyroid sickness, particularly persons with poor glycaemic control. Ischemia Modified Albumin IMAis novel marker of oxidative stress and tissue ischemia . The pathophysiological occasions of ischemia, including free oxygen radicals and hypoxia, bring about a conformational change of albumin in the $\mathrm{N}$-terminus. The study included 120 subjects, divided into four groups; the first group included 30 patients type2 diabetes mellitus T2DM with newly diagnosed hyperthyroidism HprT, the second group includes 30 patients T2DMwith newly diagnosed hypothyroidism HpoT, the third group included 30 patients diagnosed with T2DM, and the fourth group included 30 healthy control subjects. The concentration of (fasting blood glucose FBG, Glycated hemoglobin HbA1c,Total cholesterol TC, Triglyceride TG,high density lipoprotein HDL, Free Triiodothyronin FT3,Free Thyroxin FT4 and thyroid-stimulating hormone TSH). The results show high significant differences in level of IMA between all studied groups that including diabetic patient with newlydiagnosed hyperthyroidism, diabetic

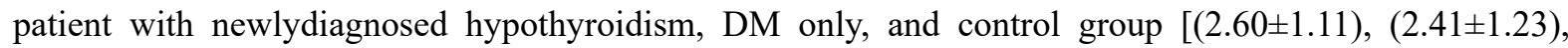
(2.57 \pm 5.50$),(2.10 \pm 0.61)]$ respectively.
\end{abstract}

Conclusion: Biomarkers IMA could be helpful early diagnosis in monitoring T2DM with newlydiagnosed thyroiddisorders.

Keywords: Type2 DM,thyroid dysfunction, ischemia modified albumin.

\section{Introduction}

Diabetes mellitus DM is a chronic endocrine issue described by hyperglycemia, which happens because of inadequacy secretion of insulin from pancreas or insulin sensitivity in the body or both of them ${ }^{(1)}$. The thyroid gland is the first endocrine gland to develop in humans ${ }^{(2)}$. Thyroid keep released growth hormone, skeletal development, and heart rate. It advances central nervous system development and invigorates synthesis of numerous enzymes, Thyroid is essential for muscle tone and life. Furthermore, metabolism is managed by the thyroxine hormone, which can be made by the thyroid if enough organic iodine is accessible ${ }^{(3)}$. Diabetic patients are at increased danger of thyroid sickness, particularly persons with poor glycaemic control ${ }^{(4)}$.
In the mid-1990s, it was first found that presentation to ischemic tissue changes the $\mathrm{N}$-terminus of the albumin serum, diminishing its coupling limit with regards to metals, (for example, cobalt, nickel, and copper) and bringing about the development of IMA ${ }^{(5)}$. Many studies used the new biochemical indicator IMA for the finding and evaluation of myocardial ischemia. In DM, both of oxidative stress and hyperglycemia will promote chronic ischemia which may lead to necrosis of several tissues and lead to different diabetic complications ${ }^{(6)}$.

\section{Material and Method}

The study included 120 subjects, divided into four groups; the first group included 30 patients with T2DM with newly diagnosed of hyperthyroidism HprT, the second group includes 30 patients with T2DMnewly 
diagnosed of hypothyroidism HpoT, the third group included 30 patients diagnosed with T2DM, and the fourth group included 30 healthy control subjects. The study was approved by the scientific and ethics committee in the national diabetes center/Al-mustansiriyah University was enrolled in the study which was conducted from January 2020 to July 2020. Blood sample was divided into two parts, the first one $(1 \mathrm{ml})$ was transferred into tube containing (EDTA), to estimate $\mathrm{HbA1C}$. While the second one $(9 \mathrm{ml})$ was transferred into a gel tube. Then centrifugation at (3000rpm) for 15 minutes to separate the serum. $1 \mathrm{ml}$ of serum was used to determine FBG and lipid profile and $1.5 \mathrm{ml}$ of serum used for further investigation thyroid function test (FT3, FT4 and TSH). The remained was transferred to the eppendorff tube and stored in a deep freezer $\left(-20^{\circ} \mathrm{C}\right)$ to be used for IMA. All biochemistry measurement were done using kenza 240TX (Biolabo) instrument and (Biolabo) kit (FBG, TC,TG, HDL) .HbA1c were measuredusing the Tosoh automated glycohemoglobin analyzer HLC-723GX. The HLC-723GX was based on the high-performance liquid chromatography HPLC. The Thyroid hormones assay(TSH,FT4 and FT3) were performed using Vidas Instruments and Biomerieux kit. The concentration of IMA determined using ELISA kit (Al-Shkairate establishments, Jordan).

\section{Result and Discussion}

As shown in table (1), the mean duration increase in diabetic with newly diagnosed hypothyroidism group HpoT and in diabetic with newlydiagnosed hyperthyroidism group HprT, as compared to the patient with DM only. But that increase was not significant. The result in the study showed the female number increase in diabetic with newly diagnosed hypothyroidism group HpoT $(27(90 \%))$ more than males $3(10 \%)$, butin diabetic with newly diagnosed hyperthyroidism group HprT, the female were $20(66.7 \%)$ and male were 10 $(33.3 \%)$.

The level of FBG showed a highly significant $(p<0.001)$ increase in diabetic with newly diagnosed hyperthyroidism group, DM only group, diabetic with newly diagnosed hypothyroidism group,and control group. The level of HbA1c showed a highly significant $(p<0.001)$ increase in diabetic with newly diagnosed hyperthyroidism group,diabetic with newly diagnosed hypothyroidism group, DM only group,and control group.

Table 1: Assessment of demographic data

\begin{tabular}{|c|c|c|c|c|c|}
\hline Variables & HprT & НроТ & Control & DM only & p-value \\
\hline Number & 30 & 30 & 30 & 30 & - \\
\hline Age years & $52.9 \pm 6.0$ & $55.7 \pm 7.0$ & $49.2 \pm 7.4$ & $53.5 \pm 7.9$ & $0.006[\mathrm{~S}]$ \\
\hline Duration Years & $6.0 \pm 4.8$ & $6.9 \pm 5.2$ & $5.7 \pm 4.4$ & - & 0.621 \\
\hline \multicolumn{6}{|l|}{ Gender } \\
\hline Female & $20(66.7 \%)$ & $27(90 \%)$ & $27(90 \%)$ & $26(86.7 \%)$ & \\
\hline Male & $10(33.3 \%)$ & $3(10 \%)$ & $3(10 \%)$ & $4(13.3 \%)$ & \\
\hline FBS mg/dl & $220.3 \pm 75.5$ & $187.3 \pm 77.1$ & $85.7 \pm 6.8$ & $196.3 \pm 102.9$ & $<0.001$ \\
\hline HbA1c \% & $8.8 \pm 0.7$ & $8.5 \pm 0.8$ & $4.8 \pm 0.4$ & $8.3 \pm 0.8$ & $<0.001$ \\
\hline
\end{tabular}

Diabetes with newly hypothyroidism HpoT: Diabetes with newly diagnosed hypothyroidism HprT: Diabetes with newly diagnosedhyperthyroidism

$\mathrm{p}$-value $<0.05$ is significant

Advanced age considered a well-known risk factor for hypothyroidism, coupled with female gender, and autoimmune disease ${ }^{(7)}$. Because of the insidious and silent nature of the disease "The American Thyroid Association" had recommended to start the screening at the age above 35 years, to be performed periodically every 5 years, for early detection of $\mathrm{HpoT}^{(8)}$. Other epidemiological study indicated that older populations are at increased risk ${ }^{(9)}$. Diabetes duration has been found to be a significant risk factor in this study sample for more than 6 years, which is not the case in studies of various ethnic groups such as the Saudi diabetic patients 
were 10 years, for Chinese 8.3 years and for Spanish population was 9.6 years $^{(10)}$.

In the current research, the incidence of thyroid conditions was higher in females than in males. These findings are in agreement with studies of Papazafiropoulou A.et al. and Aljabri KS.et al., ${ }^{(1,12)}$, founds female gender is affected by the prevalence of thyroid abnormalities in diabetic patients .In hyperthyroid with diabetes patient group, the increase level of FBG may be due to increased metabolic rate. And in hypothyroid with diabetes patient group, low FBG may be attributed to decrease metabolic rate ${ }^{(13)}$. There is a decline in insulin secretion by beta cells caused low glucose in hypothyroidism, and the reaction of beta cells to glucose is increased in hyperthyroidism due to increased beta cell mass. ${ }^{(14)}$.
The result of the present study as shown in figure (1) and (2), demonstrated a highly significant increase in the TC and TGin diabetic with newly diagnosed hypothyroidism group, and DM only group. Whereas, there is lower level of TC and TG indiabetic with newly diagnosed hyperthyroidism group, and control group. While there is a highly significant increase in the HDL in control group. And lower indiabetic with newly diagnosed hyperthyroidism group,in diabetic with newlydiagnosed hypothyroidism group, and DM only group as shown in figure (3).

The results of the present study were close to the Kumar, et al. ${ }^{(15)}$, study found that the meanTC and LDLcholesterol levels were elevated in hypothyroidism compared to the controls. Thyroid dysfunction was highly common in patients with impaired glycemic regulation, a longer duration of diabetes, and was associated with substantially higher serum cholesterol and triglyceride levels ${ }^{(16)}$.

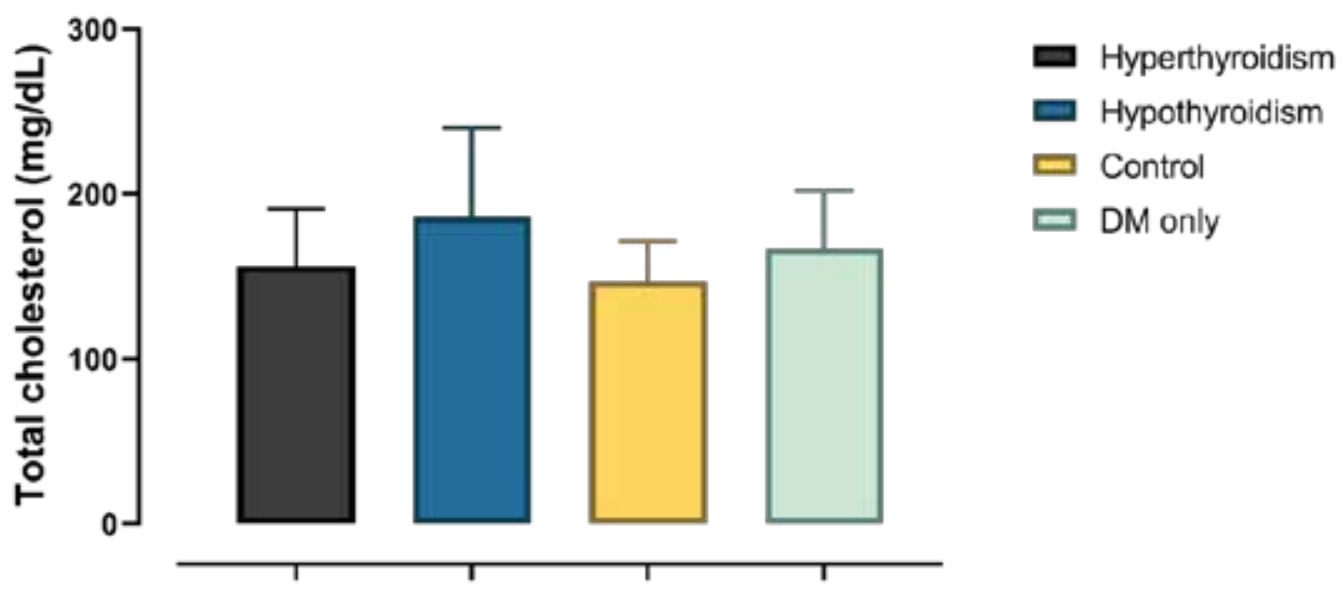

Figure 1: Assessment of total cholesterol in various groups

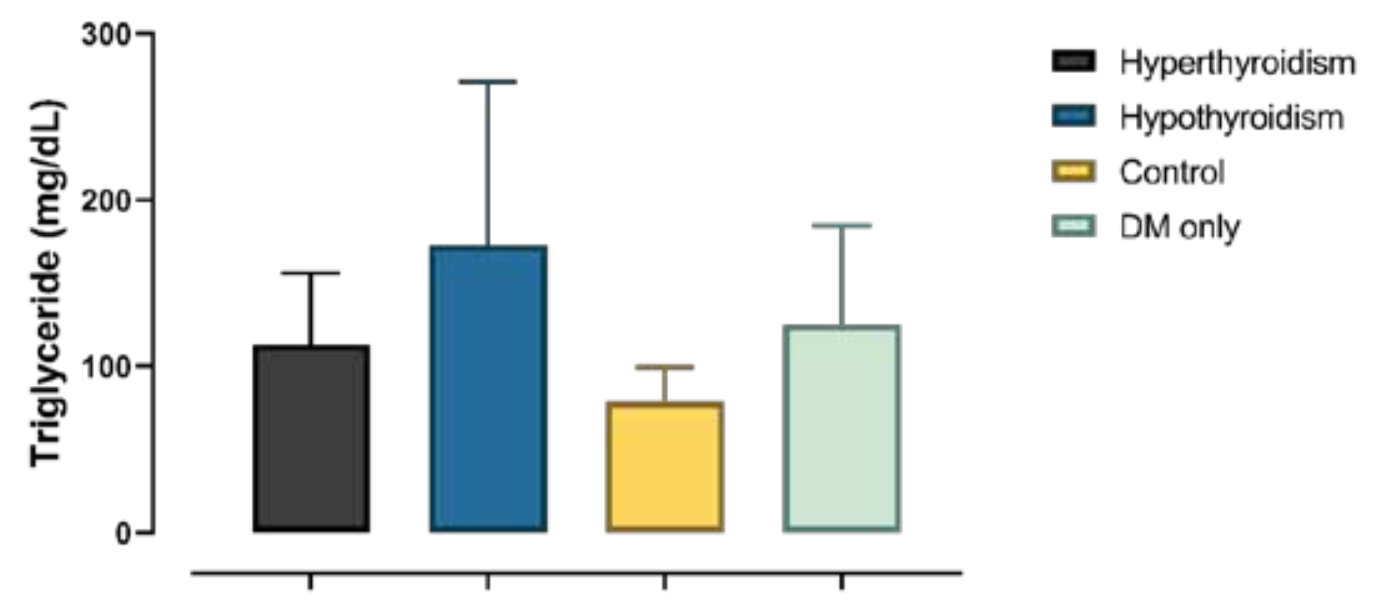

Figure 2: Assessment of triglyceride in various groups 


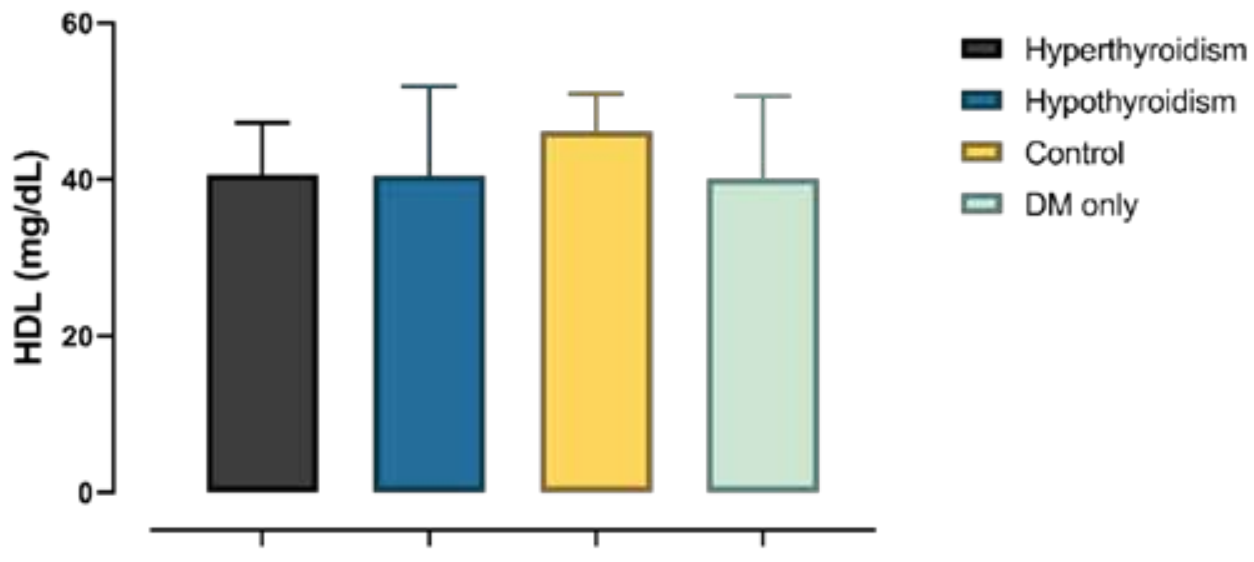

Figure 3: Assessment of HDL in various groups

Table (2), demonstrated a highly significant $(p<0.001)$ increase in the means of FT3and FT4 in diabetic with newly diagnosed hyperthyroidism group. Whereas, there were lower indiabetic with newly diagnosed hypothyroidism group, DM only group, and control group. The level of TSH is highly significant $(p<0.001)$ increase in diabetic with newly diagnosed hypothyroidism group. Whereas; it is lower indiabetic with newly diagnosed hyperthyroidism group,DM only group, and control group. The results also showed a high significant differences in the level of IMA between all studied groups including diabetic patient newly diagnosed hyperthyroidism, diabetic patient newly diagnosed hypothyroidism, DM only, and control group as shown in table (2), and figure (4).

Table 2: Assessment of thyroid function parameters

\begin{tabular}{|l|c|c|c|c|c|}
\hline Variables & HprT & HpoT & Control & DM only & p-value \\
\hline Number & 30 & 30 & 30 & 30 & - \\
\hline fT3 Pmol/1 & $11.3 \pm 3.9$ & $4.0 \pm 8.7$ & $5.3 \pm 0.7$ & $4.9 \pm 0.7$ & $<0.001[S]$ \\
\hline fT4 Pmol/1 & $25.1 \pm 7.2$ & $7.8 \pm 1.7$ & $11.3 \pm 2.7$ & $10.7 \pm 1.6$ & $<0.001[S]$ \\
\hline TSH $\mu \mathrm{mol} / 1$ & $0.1 \pm 0.001$ & $20.1 \pm 19.3$ & $1.5 \pm 0.6$ & $2.1 \pm 0.9$ & $<0.001[\mathrm{~S}]$ \\
\hline Ischemia Modified Albumin $\mathrm{ng} / \mathrm{ml}$ & $2.60 \pm 1.11$ & $2.41 \pm 1.23$ & $2.10 \pm 0.61$ & $2.57 \pm 5.50$ & $<0.001[\mathrm{~S}]$ \\
\hline
\end{tabular}

$\mathrm{p}$-value $<0.05$ is significant
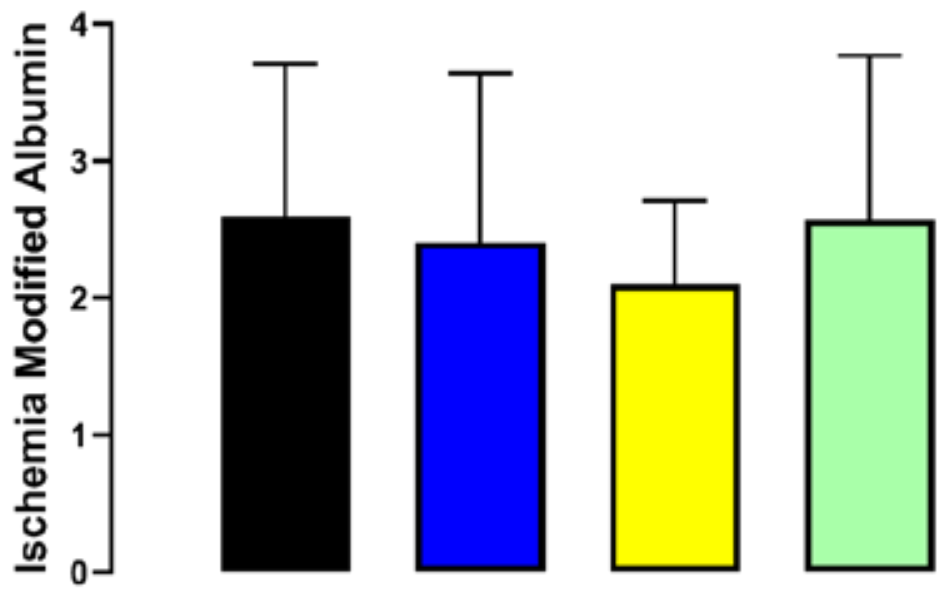

- Hyperthyroidism

Hypothyroidism

口 Control

口 DM only

Figure 4: Assessment of ischemia modified albumin in various groups 
Insulin, an anabolic hormone, is believed to increase FT4 levels while suppressing T3 levels by inhibiting T4T3 conversion in liver. In diabetes mellitus, thyrotropin releasing hormone production declines and there is also a depletion of the nocturnal TSH peak that is responsible for the incidence of low thyroid hormone levels in certain diabetics ${ }^{(17)}$. Oxidative stress (OXS) and free radicals is associated with diabetes and that leads to the onset and progression of the diabetes mellitus and occurrence of complications ${ }^{(18)}$.Many studies describe the function of IMA as a marker for ischemia induces and inflammationa cascade of proinflammatory reactions that result in reactive oxygen species ROSbeing produced ${ }^{(19)}$. It was understood that thyroid hormones control oxidative metabolis and mitochondrial respiration, and may therefore play an important role in controlling the production of free radicals and OXS. Therefore, any changes in the status of thyroid hormones can be contributed to a potential change in the status of $\mathrm{OXS}^{(20)}$. This study is agreed withMa, SG.et al.and Oncel, M.et al. ${ }^{(21,22)}$, showing a significant positive associations between ischemia modified albumin and thyroid hormones.

Ethical Clearance: The Research Ethical Committee at scientific research by ethical approval of both MOH and MOHSER in Iraq.

\section{Conflict of Interest: None}

Funding: Self-funding

\section{Reference}

1. Ishii H, Shuichi S, Williams P, Demiya S, Aranishi $\mathrm{T}$, Treuer T. Cross-sectional survey in patients with type 1 and type 2 diabetes to understand mealtime insulin unmet needs in Japan: The MINUTES-J study. Diabetes Research and Clinical Practice. 2020 Apr 1;162:108076.

2. . Benvenga S, Tuccari G, Ieni A, Vita R. Thyroid Gland: Anatomy and Physiology.2018 Elsevier. NDO2: 96022.

3. Jawad A H, Alsayed R, Ibrahim A E, Hallab Z, AlQais Z,and Yousif E. Thyroid Gland and Its Rule in Human Body. Research Journal of Pharmaceutical, Biological and Chemical. 2016. 7(6). 1336.

4. Stefanowicz-Rutkowska MM, Matuszewski W, Bandurska-Stankiewicz EM. Autoimmune Thyroid Disease is Associated with a Lower Prevalence of Diabetic Retinopathy in Patients with Type 1
Diabetic Mellitus. Medicina. 2020 Jun;56(6):255.

5. Erem C, Suleyman AK, Civan N, Mentese A, Nuhoglu I, Uzun A, Ersoz HO, Deger O. Ischemiamodified albümin and malondialdehyde levels in patients with overt and subclinical hyperthyroidism: effects of treatment on oxidative stress. Endocrine journal. 2015:EJ14-0542.

6. El Said NH, Bahgat HM, El-Fishawy HS, Hussein MA, Mohamed NA, Saleh OF. Sensitivity and specificity of ischaemia-modified albumin in detecting diabetic nephropathy. The Egyptian Journal of Internal Medicine. 2018 Oct 1;30(4):204.

7. Kim YA, Park YJ. Prevalence and risk factors of subclinical thyroid disease. Endocrinology and Metabolism. 2014 Mar 1;29(1):20-9.

8. Ladenson PW, Singer PA, Ain KB, Bagchi N, Bigos ST, Levy EG, Smith SA, Daniels GH. American Thyroid Association guidelines for detection of thyroid dysfunction. Archives of internal medicine. 2000 Jun 12;160(11):1573-5.

9. Hollowell JG, Staehling NW, Flanders WD, Hannon WH, Gunter EW, Spencer CA, Braverman LE. Serum TSH, T4, and thyroid antibodies in the United States population (1988 to 1994): National Health and Nutrition Examination Survey (NHANES III). The Journal of Clinical Endocrinology \& Metabolism. 2002 Feb 1;87(2):489-99.

10. Al-Geffari M, Ahmad NA, Al-Sharqawi AH, Youssef AM, AlNaqeb D, Al-Rubeaan K. Risk factors for thyroid dysfunction among type 2 diabetic patients in a highly diabetes mellitus prevalent society. International journal of endocrinology. 2013 Jan 1;2013.

11. Papazafiropoulou A, Sotiropoulos A, Kokolaki A, Kardara M, Stamataki P, Pappas S. Prevalence of thyroid dysfunction among greek type 2 diabetic patients attending an outpatient clinic. Journal of clinical medicine research. 2010 Apr;2(2):75.

12. Aljabri KS, Bokhari SA, Alshareef MA, Khan PM, Mallosho AM, AbuElsaoud HM, Jalal MM, Safwat RF, El Boraie R, Aljabri NK, Aljabri BK. The prevalence of hypothyroidism in patients with type 2 diabetes mellitus in Saudi community based hospital: a retrospective single centre study. Archives of diabetes \& obesity 2019; 2 (1): 104. 2018;108.

13. Kadiyala R, Peter R, Okosieme OE. Thyroid dysfunction in patients with diabetes: clinical 
implications and screening strategies. International journal of clinical practice. $2010 \mathrm{Jul} ; 64(8): 1130-9$.

14. Eisa AH, Bashir AA, Osman AA, Ali IA, Awad KM, Hajo EY, Taha EH, Alborai AA, Musa OA. Thyroid hormones, blood glucose and glycosylated hemoglobin in healthy Sudanese subjects. International Journal of Advances in Medicine. 2019 May;6(3):867.

15. Kumar A, Gupta S, Nimbalkar V. Dydlipidemia in thyroide disorders. International Journal of Medical and Biomedical Studies. 2020 Feb 18;4(2).

16. Elgazar EH, Esheba NE, Shalaby SA, Mohamed WF. Thyroid dysfunction prevalence and relation to glycemic control in patients with type 2 diabetes mellitus. Diabetes \& Metabolic Syndrome: Clinical Research \& Reviews. 2019 Jul 1;13(4):2513-7.

17. Uppal V, Vij C, Bedi GK, Vij A, Banerjee BD. Thyroid disorders in patients of type 2 diabetes mellitus. Indian Journal of Clinical Biochemistry. 2013 Oct 1;28(4):336-41.

18. Kalayci M, Cetinkaya E, Yigit K, Sabaner MC, Duman R, Balik AR, Erel Ö. Ischemia Modified

Albumin Levels and Thiol-Disulphide Homeostasis in Diabetic Macular Edema in Patients with Diabetes Mellitus Type 2. Current Eye Research. 2020 Sep 5.

19. Pande AP, Shenoy VB, Datta D, Khan NG, Ramya KR. A correlative study of serum ischemia modified albumin and total thiol in prediabetes and diabetes mellitus. International Journal of Clinical Biochemistry and Research. 2019;6(3):380-3.

20. Ersoy K, Anaforoğlu İ, Algün E. Serum ischemic modified albumin levels might not be a marker of oxidative stress in patients with hypothyroidism. Endocrine. 2013 Apr 1;43(2):430-3.

21. Ma SG, Yang LX, Bai F, Xu W, Hong B. Ischemiamodified albumin in patients with hyperthyroidism and hypothyroidism. European journal of internal medicine. 2012 Sep 1;23(6):e136-40.

22. Oncel M, Kiyıc1 A, Onen S. Evaluation of the relationship between ischemia-modified albumin levels and thyroid hormone levels. Journal of clinical laboratory analysis. 2015 Nov;29(6):427431. 